Pacific Journal of Mathematics

GROWTH ESTIMATES FOR THE SINGULAR VALUES O 


\section{GROWTH ESTIMATES FOR THE SINGULAR VALUES OF SQUARE-INTEGRABLE KERNELS}

\section{James Alan Cochran}

This paper is concerned with the unique correspondences which exist between the values of convergence exponents for the classical Fourier coefficients of one-variable functions satisfying various smoothness assumptions, on the one hand, and growth estimates for the singular values $\mu_{n}$ associated with square-integrable two-variable kernels $K(x, y), a \leqq x, y \leqq b$, having comparable smoothness, on the other. Extending earlier work of the author and others, precise values are given for the infimum of $\gamma$ for which $\Sigma\left(1 / \mu_{n}\right)^{\gamma}$ converges when $K$ satisfies Lipschitz conditions, integrated Lipschitz conditions, is of bound variation, or a combination of these.

1. Introduction. In this paper we shall be concerned with $\mathscr{L}^{2}$ kernels, i.e. two-variable functions $K(x, y), a \leqq x, y \leqq b$, satisfying

$$
\|K\| \equiv\left[\int_{a}^{b} \int_{a}^{b}|K(x, y)|^{2} d x d y\right]^{1 / 2}<\infty
$$

and their singular values $\mu_{n}$. We take the $\mu_{n}$ to be the nonnegative square roots of the characteristic values of the nonnegative definite kernel $K K^{*}(x, y)$, and thus

$$
\phi_{n}(x)=\mu_{n}^{2} \int_{a}^{b} \int_{a}^{b} K(x, z) \overline{K(y, z)} \phi_{n}(y) d y d z
$$

with $\left\|\phi_{n}\right\| \neq 0$. Our interest is in the growth behavior of these singular values as a function of the smoothness of the given kernel $K$.

Our point of departure is the knowledge that the best mean square approximation to a given square-integrable kernel $K$ by degenerate kernels of the form

$$
K_{N}(x, y)=\sum_{n=1}^{N} a_{n}(x) \bar{b}_{n}(y) \quad a_{n}, b_{n} \in \mathscr{L}^{2}
$$

occurs, for fixed $N$, when the $a_{n}, b_{n}$ are proportional to the singular functions $\phi_{n}, \Psi_{n}$ of $K$ [9]. To be precise, it turns out that 


$$
\begin{aligned}
\left\|K(x, y)-\sum_{n=1}^{N} a_{n}(x) \bar{b}_{n}(y)\right\|^{2} & \geqq\left\|K(x, y)-\sum_{n=1}^{N} \frac{\phi_{n}(x) \bar{\Psi}_{n}(y)}{\mu_{n}}\right\|^{2} \\
& =\|K\|^{2}-\sum_{n=1}^{N}\left(\frac{1}{\mu_{n}}\right)^{2} \\
& =\sum_{n=N+1}^{\infty}\left(\frac{1}{\mu_{n}}\right)^{2},
\end{aligned}
$$

where we have assumed that the singular functions are orthonormalized and then used the fact that [3], [9]

$$
\sum_{n}\left(\frac{1}{\mu_{n}}\right)^{2}=\|K\|^{2}
$$

In the special case when $a=0, b=\pi$, the $a_{n}$, say, are the appropriate normalized trigonometric functions, and the $\bar{b}_{n}$ then are the resulting Fourier coefficients of $K(x, y)$ viewed as a function of $x$ alone, (1.2) takes the form

$$
\sum_{n=N+1}^{\infty}\left(\frac{1}{\mu_{n}}\right)^{2} \leqq\|K\|^{2}-\sum_{n=1}^{N} \int_{0}^{\pi}\left|b_{n}(y)\right|^{2} d y
$$

In fact, using Parseval's relation, the right-hand side of the inequality can be further rewritten as

$$
\sum_{n=N+1}^{\infty}\left(\frac{1}{\mu_{n}}\right)^{2} \leqq \sum_{n=N+1}^{\infty} \int_{0}^{\pi}\left|b_{n}(y)\right|^{2} d y
$$

This last expression shows how convergence exponents for the trigonometric Fourier coefficients of one-variable functions satisfying various smoothness assumptions can be used to generate growth estimates for the singular values associated with two-variable kernels having comparable smoothness with respect to one of the two variables. These estimates, moreover, are sharp since for difference kernels the singular values and the related Fourier coefficients are essentially reciprocals.

In the next section of this paper we set down the requisite mathematical details needed for our investigation. Subsequently, in $\S 3$, we discuss the spectral-theoretic results associated with the various kernel smoothness conditions of interest. Typical of the results obtained there is essentially the following:

THEOREM 3.3. If $\partial^{s} K(x, y) / \partial x^{s}$ is relatively uniformly of bounded variation (in $x$ ) for some nonnegative integer $s$, and also satisfies an 
integrated Lipschitz condition (in $x$ ) of order $q \geqq 1$ with exponent $\beta>0$, then the series of reciprocal singular values is $\gamma$-summable for all $\gamma>\rho$ where

$$
\rho= \begin{cases}\frac{1}{1+s} & \beta q<1 \\ \frac{q}{q(\beta+1+s)-1} & \beta q \geqq 1, q \leqq 2 \\ \frac{2(q-1)}{q(\beta+2+2 s)-3-2 s} & \beta q \geqq 1, q>2 .\end{cases}
$$

This particular result, which generalizes an earlier theorem of the author [4], is the precise analogue of a related Fourier series result derivable from work of Hardy and Littlewood [7]. In like manner, the other results of $\$ 3$, or their sundry special cases, have been engendered by either known or otherwise provable classical Fourier-theoretic results due principally to Bernstein, Zygmund, Hardy and Littlewood, and Szász. Comparable results can be obtained in analogous fashion for kernels on multi-dimensional domains using related work of Taibleson [12].

2. Preliminaries. For convenience, and without loss of generality, we let $a=0, b=\pi$ and consider the class of $\mathscr{L}^{2}$ kernels $K(x, y)$ with $0 \leqq x, y \leqq \pi$. We take $x$ as the variable of interest and define

$$
K^{(r)}(x, y) \equiv \frac{\partial^{r} K(x, y)}{\partial x^{r}}
$$$$
(r=0,1, \cdots, s)
$$

for nonnegative integer $s$. Assume that $K(x, y)$ is extended, as an even function of $x$ if $s$ is even, and as an odd function of $x$ if $s$ is odd, into the domain $-\pi \leqq x \leqq 0$, and thence as a periodic function of $x$ with period $2 \pi$.

We shall say that $K^{(s)}(x, y)$ relatively uniformly satisfies a Lipschitz condition with exponent $\alpha$ if

$$
\left|K^{(s)}(x+h, y)-K^{(s)}(x-h, y)\right|<|h|^{\alpha} A(y) \quad(0<\alpha \leqq 1)
$$

where $A(y)$ is nonnegative and square-integrable. If $A$ is bounded, $K^{(s)}$ satisfies a Lipschitz condition in the more classical sense. Likewise $K^{(s)}(x, y)$ is said to be relatively uniformly of bounded variation if for all $N \geqq 1$ and arbitrary choice of partition $0 \leqq x_{0} \leqq x_{1} \leqq$ $\cdots \leqq x_{N} \leqq \pi$ 


$$
\sum_{n=1}^{N}\left|K^{(s)}\left(x_{n}, y\right)-K^{(s)}\left(x_{n-1}, y\right)\right|<B(y)
$$

where $B(y)$ also is nonnegative and square-integrable. Analogous definitions prevail if the roles of $x$ and $y$ are interchanged.

More generally, we shall say that $K^{(s)}(x, y)$ relatively uniformly satisfies an integrated Lipschitz condition of order $p$ (with exponent $\alpha$ ) if

$$
\int_{0}^{\pi}\left|K^{(s)}(x+h, y)-K^{(s)}(x-h, y)\right|^{p} d x<|h|^{\alpha p} A^{p}(y)
$$

with $\mathscr{L}^{2} A \geqq 0$. For convenience, moreover, we shall use the classical terminology, namely $\operatorname{Lip} \alpha$ and $\operatorname{Lip}(\alpha, p)$ to denote the classes of $\mathscr{L}^{2}$ kernels which relatively uniformly satisfy a Lipschitz condition with exponent $\alpha$ or an integrated Lipschitz condition of order $p$, respectively.

For later reference we note (omitting the more direct proofs):

Property 1. Kernels in $\operatorname{Lip}(\alpha, p)$ also belong to Lip $(\alpha, q)$ for all $1 \leqq q<p$. Kernels in Lip $\alpha$ are automatically in Lip $(\alpha, p)$ for all $p \geqq 1$.

Property 2. Kernels which are relatively uniformly of bounded variation belong to $\operatorname{Lip}(1,1)$.

Property 3. If $K(x, y)$ is absolutely continuous in $x$, for almost all $y$, and

$$
\int_{0}^{\pi}\left[\int_{0}^{\pi}\left|K^{(1)}(x, y)\right|^{p} d x\right]^{2 / p} d y<\infty
$$

$p>1$, then $K(x, y)$ is in $\operatorname{Lip}(1, p)$.

Proof. For $h>0$,

$$
\begin{aligned}
\int_{0}^{\pi} \mid K(x+h, y) & -\left.K(x-h, y)\right|^{p} d x=\int_{0}^{\pi}\left|\int_{-h}^{h} K^{(1)}(x+t, y) d t\right|^{p} d x \\
& \leqq(2 h)^{p} \int_{0}^{\pi}\left(\frac{1}{2 h} \int_{-h}^{h}\left|K^{(1)}(x+t, y)\right| d t\right)^{p} d x \\
& \leqq(2 h)^{p} \int_{0}^{\pi} \frac{1}{2 h} \int_{-h}^{h}\left|K^{(1)}(x+t, y)\right|^{p} d t d x \\
& =(2 h)^{p} \int_{0}^{\pi}\left|K^{(1)}(x, y)\right|^{p} d x .
\end{aligned}
$$


The last equality follows from interchange of the order of integration and utilization of the periodicity properties of $K(x, y)$.

Property 4. If a kernel belongs both to $\operatorname{Lip}(\alpha, p)$ and to $\operatorname{Lip}(\beta, q)$ with $p<q$, then it belongs to Lip $(\gamma, r)$ for all $p \leqq r \leqq q$, where

$$
\gamma=\alpha \frac{p(q-r)}{r(q-p)}+\beta \frac{q(r-p)}{r(q-p)} .
$$

3. Growth estimates for singular values. The singular values associated with a given $\mathscr{L}^{2}$ kernel $K(x, y), 0 \leqq x, y \leqq \pi$, are those positive values $\mu$ for which there exist nontrivial $\phi(x), \Psi(x)$ satisfying the coupled equations

$$
\phi(x)=\mu \int_{0}^{\pi} K(x, y) \Psi(y) d y
$$

$$
\Psi(x)=\mu \int_{0}^{\pi} \overline{K(y, x)} \phi(y) d y .
$$

There are at most countably many of these, and they are customarily ordered (indexed) according to increasing size. The associated singular functions given by (3.1) can be chosen to be orthonormal amongst themselves as well as biorthogonal with respect to the kernel $K$. It then follows that

$$
K(x, y)=\sum_{n=1}^{\infty} \frac{\phi_{n}(x) \bar{\Psi}_{n}(y)}{\mu_{n}}
$$

where the series on the right-hand side of this expression is mean convergent. Moreover, the relationship (1.3) is valid.

The convergence of $\Sigma\left(1 / \mu_{n}\right)^{\gamma}$ for exponents $\gamma$ smaller than 2 can only be established under additional restrictions on the kernel $K(x, y)$. A singular conclusion of the kind we seek was in effect contained in a little-known paper of Smithies [8]. Before stating the result we make the basic assumptions that the square-integrable kernel $K(x, y)$ is such that the $K^{(r)}(x, y), 0 \leqq r \leqq s-1$, are absolutely continuous in $x$, a.e. in $y$, for some positive (nonnegative) integer $s$, and $K^{(s)}(x, y)$ is $\mathscr{L}^{p}(x)$, a.e. in $y$, for some $p>1$. Then

THEOREM 3.1. If $K^{(s)}(x, y)$ belongs to Lip $(\alpha, p), \Sigma\left(1 / \mu_{n}\right)^{\gamma}$ converges for all $\gamma>\rho$ where 


$$
\rho= \begin{cases}\frac{1}{\alpha+s+1-1 / p} & 1<p \leqq 2 \\ \frac{1}{\alpha+s+1 / 2} & p>2 .\end{cases}
$$

When $s=0$, the additional proviso $\alpha+1 / 2>1 / p$ may be needed since $K \in \mathscr{L}^{2}$.

Actually Smithies only was interested in the case $1<p \leqq 2$. The extension to $p>2$ follows readily using Property 1 .

For the proof of this theorem we essentially need only make use of a classical result of Szász [10], [11], generalized to higher-order derivatives, and employ (1.4). Special attention may have to be given to the end-points of the interval, however, and the behavior of $K^{(r)}$ appropriately modified there. Fortunately, this can be accomplished by adding to $K$ a degenerate kernel of the form (1.1), and, importantly, such a degenerate perturbation leaves unchanged the dominant asymptotic growth estimate for the singular values (Fan [5]).

It is worth mentioning that, in view of Property 3, the above theorem is more general than a result of Chang [2]. Moreover, the special case $s=0$ contains the analogue of the well-known theorem of Bernstein (see Bary [1], pp. 153-171, or Zygmund [15], pp. 240-243, for example) on absolutely convergent Fourier series.

Exercising some care, the convexity condition Property 4 may be used with Theorem 3.1 to yield

THEOREM 3.2. If $K^{(s)}(x, y)$ belongs both to Lip $(\alpha, p)$ and to Lip $(\beta, q)$, with $p<q$, then $\Sigma\left(1 / \mu_{n}\right)^{\gamma}$ converges for all $\gamma>\rho$ where

(i) for $q \leqq 2$,

$$
\rho= \begin{cases}\frac{1}{\alpha+s+1-1 / p} & p q(\alpha-\beta)>q-p \\ \frac{1}{\beta+s+1-1 / q} & p q(\alpha-\beta) \leqq q-p\end{cases}
$$

(ii) for $p \leqq 2<q$,

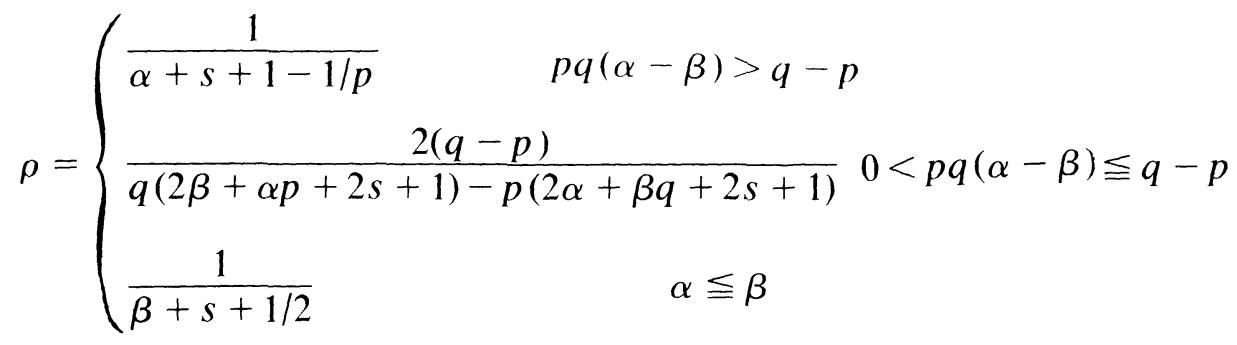


(iii) and for $p>2$,

$\rho= \begin{cases}\frac{1}{\alpha+s+1 / 2} & \alpha>\beta \\ \frac{1}{\beta+s+1 / 2} & \alpha \leqq \beta .\end{cases}$

When $s=0$, the additional provisos $\alpha+1 / 2>1 / p$ and $\beta+1 / 2>1 / q$ may be needed.

Owing to Properties 1,2, as special cases we have

THEOREM 3.3. If $K^{(s)}(x, y)$ is relatively uniformly of bounded variation and also in Lip $(\beta, q)$ for some $\beta>0, q \geqq 1$, then $\Sigma\left(1 / \mu_{n}\right)^{\gamma}$ converges for all $\gamma>\rho$ where

$$
\rho= \begin{cases}\frac{1}{1+s} & \beta q<1 \\ \frac{q}{q(\beta+1+s)-1} & \beta q \geqq 1, q \leqq 2 \\ \frac{2(q-1)}{q(\beta+2+2 s)-3-2 s} & \beta q \geqq 1, q>2 ;\end{cases}
$$

and

TheOREM 3.4. If $K^{(s)}(x, y)$ belongs both to Lip $(\alpha, p)$ and to Lip $\beta$, then $\Sigma\left(1 / \mu_{n}\right)^{\gamma}$ converges for all $\gamma>\rho$ where

$$
\rho=\left\{\begin{array}{lr}
\frac{p}{p(\alpha+1+s)-1} & p(\alpha-\beta)>1, p \leqq 2 \\
\frac{2}{\beta(2-p)+\alpha p+1+2 s} & 0<p(\alpha-\beta) \leqq 1, p \leqq 2 \\
\frac{1}{\alpha+s+1 / 2} & \alpha>\beta, p>2 \\
\frac{1}{\beta+s+1 / 2} & \alpha \leqq \beta .
\end{array}\right.
$$

When $\mathrm{s}=0$, Theorem 3.2 could be established alternatively by first extending a classical Fourier series result of Hardy and Littlewood [6], [7]. Theorems 3.3, 3.4 contain the analogues of well-known Fouriertheoretic results of Zygmund [13], [14], [15]. 
In view of the Weyl-Chang inequalities (see Cochran [3], pp. 243-245, for example) all of the above theorems extend the known results concerning the growth behavior of the characteristic values of "smooth" kernels and settle an earlier conjecture of the author ([3], p. 266).

\section{REFERENCES}

1. N. K. Bary, A treatise on trigonometric series, Vol. II (translated from the 1961 Russian ed. by M. F. Mullins), Pergamon, New York, 1964.

2. S. H. Chang, A generalization of a theorem of Hille and Tamarkin with applications, Froc. London Math. Soc., (3) 2 (1952), 22-29.

3. J. A. Cochran, The Analysis of Linear Integral Equations, McGraw-Hill, New York, 1972.

4. - The nuclearity of operators generated by Hölder continuous kernels, Proc. Cambridge Phil. Soc., 75 (1974), 351-356.

5. K. Fan, Maximum properties and inequalities for the eigenvalues of completely continuous operators, Proc. Nat. Acad. Sci. U.S.A., 37 (1951), 760-766.

6. G. H. Hardy and J. E. Littlewood, A convergence criterion for Fourier series, Math. Zeit, 28 (1928), 612-634; see also Collected papers of G. H. Hardy,Vol. III, Clarendon. Oxford, 1969, pp. 28-51.

7. Notes on the theory of series (IX): On the absolute convergence of Fourier series, J. London Math. Soc., 3 (1928), 250-253; see also Collected papers of G. H. Hardy, op. cit., 1969, pp. 52-56.

8. F. Smithies, The eigen-values and singular values of integral equations, Proc. London Math. Soc., (2) 43 (1937), 255-279.

9. — Integral Equations, Cambridge, London, 1962, pp. 148-150.

10. O. Szász, Über den Konvergenzexponenten der Fourierschen Reihen gewisser Funktionenklassen, Sitzungsber. Akad. Wiss. München Math. Phys. KI. (1922), 135-150; see also Collected mathematical papers, Dept. of Math., Cincinnati, Cincinnati, Ohio, 1955, pp. 684-699.

11. —_ Über diz Fourierschen Reihen gewisser Funktionenklassen, Math. Ann., 100 (1928), 530-536; see also Collected mathematical papers, op. cit., 1955, pp. 758-764.

12. M. H. Taibleson, On the theory of Lipschitz spaces of distributions on euclidean n-space - I, II, III, J. Math. Mech., 13 (1964), 407-479; 14 (1965), 821-839; 15 (1966), 973-981.

13. A. Zygmund, Remarque sur la convergence absolue des séries de Fourier, J. London Math. Soc., 3 (1928), 194-196.

14. - Some points in the theory of trigonometric and power series, Trans. Amer. Math. Soc., 36 (1934), 586-617.

15. — Trigonometric Series, Vols. I, II, University Press, Cambridge, 1968.

Received November 3, 1973.

Virginia Polytechnic Institute AND State University 



\section{Pacific Journal of Mathematics}

\section{Vol. 56, No. $1 \quad$ November, 1975}

Shimshon A. Amitsur, Central embeddings in semi-simple rings .......... 1

David Marion Arnold and Charles Estep Murley, Abelian groups, A, such

that $\operatorname{Hom}(A,---)$ preserves direct sums of copies of $A \ldots \ldots \ldots .$.

Martin Bartelt, An integral representation for strictly continuous linear

operators ................................... 21

Richard G. Burton, Fractional elements in multiplicative lattices......... 35

James Alan Cochran, Growth estimates for the singular values of

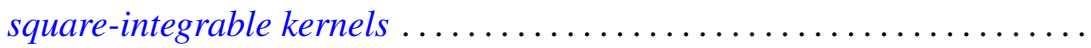

C. Martin Edwards and Peter John Stacey, On group algebras of central

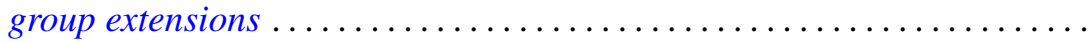

Peter Fletcher and Pei Liu, Topologies compatible with homeomorphism

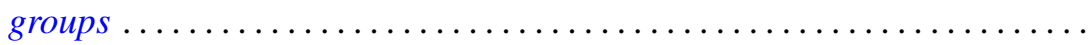

George Gasper, Jr., Products of terminating ${ }_{3} F_{2}(1)$ series ............ 87

Leon Gerber, The orthocentric simplex as an extreme simplex ............

Burrell Washington Helton, A product integral solution of a Riccati

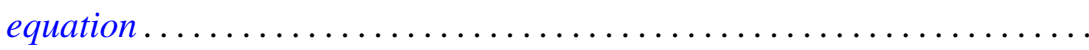

Melvyn W. Jeter, On the extremal elements of the convex cone of

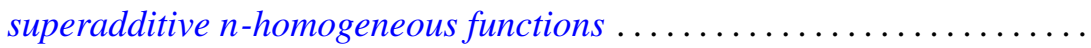

R. H. Johnson, Simple separable graphs .

Margaret Humm Kleinfeld, More on a generalization of commutative and

alternative rings. . .

A. Y. W. Lau, The boundary of a semilattice on an $n$-cell.

Robert F. Lax, The local rigidity of the moduli scheme for curves ...

Glenn Richard Luecke, A note on quasidiagonal and quasitriangular

operators .

Paul Milnes, On the extension of continuous and almost periodic functions

Hidegoro Nakano and Kazumi Nakano, Connector theory.

James Michael Osterburg, Completely outer Galois theory of perfect rings ..................................

Lavon Barry Page, Compact Hankel operators and the F. and M. Riesz

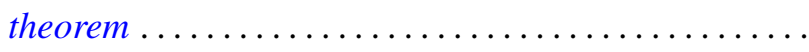

Joseph E. Quinn, Intermediate Riesz spaces..................... 225

Shlomo Vinner, Model-completeness in a first order language with a generalized quantifier.

Jorge Viola-Prioli, On absolutely torsion-free rings ..........

Philip William Walker, A note on differential equations with all solutions of integrable-square............................

Stephen Jeffrey Willson, Equivariant maps between representation 